\title{
New insight into cancer therapeutics: Induction of differentiation by regulating the Musashi/Numb/Notch pathway
}

\author{
Yoshinori Nishimoto $^{1}$, Hideyuki Okano ${ }^{1}$ \\ ${ }^{1}$ Department of Physiology, School of Medicine, Keio University, 35 Shinanomachi, Shinjuku, Tokyo 160-8582, Japan \\ Cell Research (2010) 20:1083-1085. doi:10.1038/cr.2010.122; published online 31 August 2010
}

The Musashi (Msi) family is a group of RNA-binding proteins characterized by two RNA recognition motifs (RRMs) and is evolutionarily conserved $[1,2]$. In mammals, two isoforms of this family, Msi1 and Msi2, are co-expressed in neural precursor cells, including neural stem cells (NSCs). Msi2 exhibits high sequence homology with Msil, which is more than $90 \%$ at the amino acid level within the RNA-binding domain. Msi2 is transcribed over the tissues ubiquitously [3] in contrast with Msi1 enrichment in neural stem cells or progenitor cells of the peri-ventricular area in the embryonic [4] and postnatal [5] mammalian brains. When both Msi1 and Msi2 genes were knocked-out, neurosphere formation was markedly inhibited; however, single knockout did not bring about such disturbance [6].

Originally, Msi was identified as a required factor for the asymmetric cell division of the sensory organ precursor cell (SOP) of the Drosophila adult external sensory organ [7]. In mammals, the function of Msi has been, thus far, found to activate Notch signaling through the translational repression of NUMB, which represses an intracel-

Correspondence: Hideyuki Okano

Tel: +81-3-5363-3747; Fax: +81-3-3357-5445

E-mail: hidokano@sc.itc.keio.ac.jp,hidokano@ a2.keio.jp lular Notch signaling, by binding to the $3^{\prime}$ untranslated region (UTR) of the Numb mRNA [8]. Activation of Notch signaling induces the transactivation of the promoter of the Hes- 1 gene (Figure 1). The activation of Notch-pathway is known to regulate the self-renewal of NSCs positively [9]. Previously, our laboratory reported that Msi1 and Msi2 developmentally controlled selfrenewal of embryonic cells, as well as of adult CNS stem/progenitor cells [6]. Based on the evidence that tissue stem cells exist in many adult tissues, including the hematopoietic system, intestine, mammary gland, testis, skeletal muscle, skin, hair follicle and myocardium, other than the CNS and neural crest-derived tissue, Msi/Numb/Notch signaling is considered to be associated with many adult malignancies [2]. In fact, not only at the normal developmental stages, the Msi-signaling pathway is also reported to work during tumorigenesis in several adult tissues, including glioblastoma and esophageal, colon, pulmonary, mammary and bladder carcinomas [2, 10-13]. Intriguingly, in the case of esophageal adenocarcinoma, Msil expression was the highest in glands during early cancer development. In contrast, Msil level become weaker when esophageal adenocarcinoma grows into the advanced stage. Such findings have advocated a concept of "cancer stem cells" which expands to some kinds of solid tumors [10]. Then, Msil could be a versatile marker of "cancer stem cells" especially during an early phase with strong proliferative activities. This suggests the possibility that Msi1 might have some important roles as a trigger of proliferative switching from differentiated state to cancer production in a reverse direction to development.

Although a previous report showed that Msi2 gene was rearranged to form a Msi2/HoxA9 fusion gene in a chronic myeloid leukemia (CML) case with the $7 \mathrm{p} 15$ breakpoint [14], the recent papers reported by Ito et al. [12] and Kharas et al. [13] were the first reports to show clearly the relationship between Msi2-inducing pathway and hematopoietic malignancy. These two reports suggested a new strategy for the therapy of aggressive leukemia. Using the in vivo models of CML in a chronic phase and in an aggressive blast phase [15], it was proposed that Msi2-Numb pathway should control the differentiation of CML cells and that this pathway could be the novel target of leukemia treatment [12].

Ito et al. first asked whether the levels of MSI/NUMB expression and Notch signaling altered in the CML model mice [12]. Myeloid blast crisis was modeled by transplanting hematopoietic stem cells (HSCs)-enriched KLS cells, 
which was transduced with $B c r-A b l$ and Nup98-HoxA9. HOXA9 regulates expression of many genes that are implicated in hematopoiesis and in tumorigenesis. Using the established mouse model representing chronic phase and myeloid blast crisis CML (initiated by the $B c r-A b l$ translocation and progressed by Nup98-HoxA9 expression) [16], they found Numb was expressed at significantly lower levels and Msi2 was particularly elevated in the blast crisis phase. Notably, Msi2 was expressed at higher levels than Msi 1 in the CML mice model, especially in hematopoietic stem cell-enriched populations including KLS cells and the lineage-negative fraction of blast crisis CML. Moreover, they identified a putative HoxA9-binding element at the position $-5.7 \mathrm{~kb}$ in the murine
Msi2 gene. Nup98-HoxA9 transduced with retroviral vector along with $B c r$ Abl could overexpress the Msi2 gene in KLS cells and upregulation of MSI2 expression led to downregulation of NUMB (Figure 1). At the same time, Kharas et al. established doxycyclineinducible Msi2 expression systems both in vitro and in vivo, which culminated in hematopoietic colony-forming with more immature myeloid phenotype in vitro and expansion of hematopoietic stem cells (HSCs) and short-term progenitor cells in vivo [13]. They also transduced doxycycline-inducible Msi2 cells with the $B c r-A b l$ oncogene and transplanted them into mice. Consistent with the other report, Msi2 induced immature myeloid leukemia that mimicked CML blastic crisis phase. Conversely, when Msi2 was knocked down, an in-

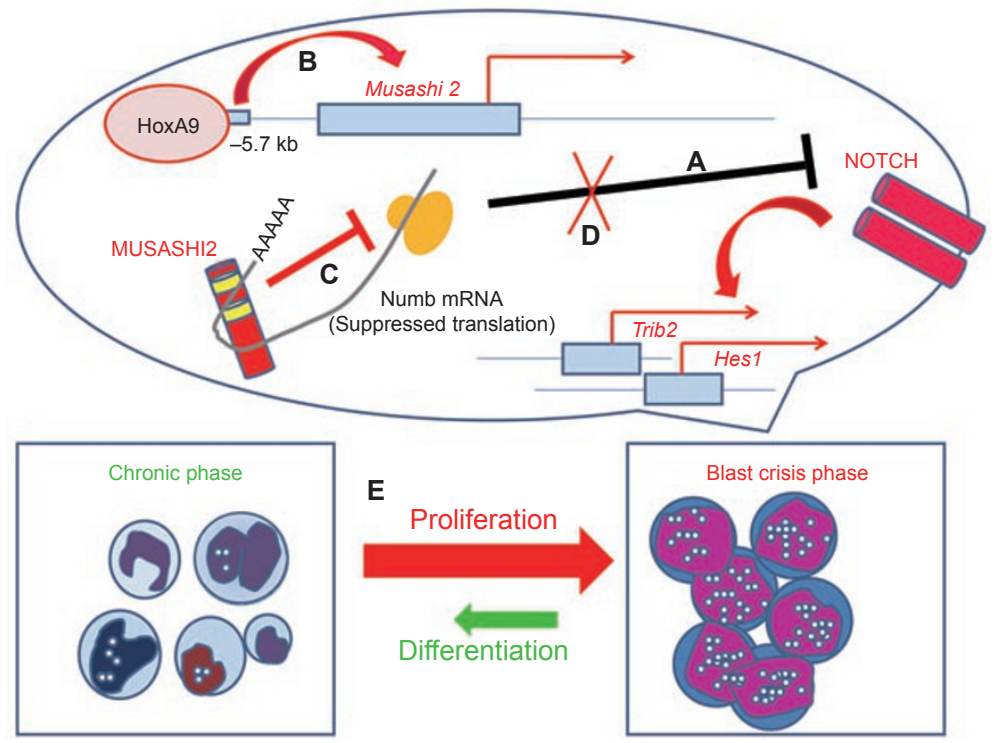

Figure 1 Scheme of the CML state in an aggressive blast crisis phase. In chronic phase, Numb blocks activation of the Notch signaling (A). During human CML progression, the following changes occurs; Nup98-HoxA9 oncogene binds the putative element at $5.7 \mathrm{~kb}$ upstream of transcription start site to trigger the upregulated expression of Musashi2 gene (B). The elevated level of MUSASHI2 leads to the downregulation of NUMB, by binding to the $3^{\prime}$ UTR of Numb mRNA to inhibit translation (C). Inhibition of Notch signaling is cancelled by suppression of NUMB (D), leading to the elevated expression of the targets, Hes1 gene and Trib2 gene. A series of these activation cause proliferative change from chronic phase to blast crisis phase of CML (E). Red symbols and letters represent increased level of each factor and enhanced activation (or inhibition) in blast crisis phase CML.

creased amount of NUMB protein in the LAMA-84 cell lines, derived from the blast crisis CML patient, was observed with immunoblot.

Then, Ito et al. asked whether human leukemia in blast crisis showed aberrant up-regulation of Msi2 as well. Analysis of samples from 30 Korean and British patients showed significant higher levels of Msi2 gene expression in blast crisis CML. As predicted from the CML mice model, microarray data of 90 samples from banks in the United States showed elevated levels of Msi2, HoxA9 and Hes1 and decrement of Numb among most of CML patients in the blast crisis phase. Similarly, Kharas et al. [13] showed the increased level of Msi2 and decreased level of Numb from 33 samples of myeloid blast crisis by comparing with 57 samples of chronic phase CML obtained in the United States.

It is particularly noteworthy that Ito et al. suggested the apparent clues suppressing tumor growth and mortality rates in the established CML model mice in vivo. They showed that a higher Numb expression made leukemia more differentiated and unable to propagate disease markedly. In fact, transplantation of cells expressing Numb decreased the frequency of developing leukemia in the model mice, from $83 \%$ to $63 \%$. When the authors transplanted cells transfected with Numb, from primary transplanted mice for donor-derived cells, into irradiated recipient secondary mice, the survival rate to evaluate propagation of disease was elevated compared to the control, from $20 \%$ to $93 \%$. These results showed that the elevated levels of NUMB can inhibit disease progression. Additionally, using gene-trap mutants or short hairpin RNA approach, loss of Msi2 impaired leukemia growth and increased survival rates significantly in vivo, especially in blast crisis CML. Similar to NUMB induction, Msi2-shRNA induced the differentiation of leukemic cells and inhibited their propagating ability [12]. 
Ito et al. also reported the association of Msi2 with high risk of relapse after allogeneic transplantation and poorer outcomes, indicating the possibility to make use of MSI2 as an early marker of advanced CML. On the other hand, Kharas et al. confirmed that Msi2 expression had relevance to poor clinical prognosis among at least congenitally normal AML subjects. Moreover, among them, they showed that the population, whose gene signatures were negatively correlated with genes altered by the Msi2-knockdown, was associated with poor prognosis. Using multivariate analyses with numbers of Msi2-associated factors, we might predict the prognosis or definitive diagnosis more accurately.

Novel findings about the Msi2/ Numb/Notch signaling pathway described above will shed light on a key to understanding development of hematologic malignancies including blast crisis phase CML. Other recent papers have revealed the relationship between Notch signaling/Hes1 and CML progression [17]. Together with these findings, each of Msi family could be a key regulator of several intracellular pathways, deciding self-renewal of the stem cells, tumorigenesis and differentiation, which is cell-fate exactly.

Further studies about relationship between Msi-signaling pathway and malignancies will be investigated. Then, differentiation induction into mature asymmetric cells by modulating the pathway in cancer cells is expected to be the novel therapeutics for advanced leukemia and other solid carcinomas.

\section{References}

1 Okano H, Imai T, Okabe M. Musashi: a translational regulator of cell fates. $J$ Cell Sci 2002; 115(Pt 7):1355-1359.

2 Okano H, Kawahara H, Toriya M, Nakao K, Shibata S, Imai T. Function of RNA-binding protein Musashi-1 in stem cells. Exp Cell Res 2005; 306:349356.

3 Sakakibara S, Nakamura Y, Satoh $\mathrm{H}$, Okano H. Rna-binding protein Musashi2. Developmentally regulated expression in neural precursor cells and subpopulations of neurons in mammalian CNS. J Neurosci 2001; 21:80918107.

4 Sakakibara S, Imai T, Hamaguchi K, et al. Mouse-Musashi-1, a neural RNAbinding protein highly enriched in the mammalian CNS stem cell. Dev Biol 1996; 176: 230-242.

5 Sakakibara S, Okano H. Expression of neural RNA-binding proteins in the postnatal CNS: implications of their roles in neuronal and glial cell development. J Neurosci 1997; 17:8300-8312.

6 Sakakibara S, Nakamura Y, Yoshida T, et al. RNA-binding protein Musashi family: roles for CNS stem cells and a subpopulation of ependymal cells revealed by targeted disruption and antisense ablation. Proc Natl Acad Sci USA 2002; 99:15194-15199.

7 Nakamura M, Okano H, Blendy JA, Montell C. Musashi: a neural RNAbinding protein required for Drosophila adult external sensory organ development. Neuron 1994; 13:67-81.

8 Imai $\mathrm{T}$, Tokunaga $\mathrm{A}$, Yoshida $\mathrm{T}$, et al. The neural RNA-binding protein Musashil translationally regulates mammalian numb gene expression by interacting with its mRNA. Mol Cell Biol 2001; 21:3888-3900.

9 Hitoshi S, Alexson T, Tropepe V, et al.
Notch pathway molecules are essential for the maintenance, but not the generation, of mammalian neural stem cells. Genes Dev 2002; 16:846-858.

10 Bobryshev YV, Freeman AK, Botelho NK, et al. Expression of the putative stem cell marker Musashi-1 in Barrett's esophagus and esophageal adenocarcinoma. Dis Esophagus 2010 Apr 29. doi: 10.1111/j.1442-2050.2010.01061.x

11 Todaro M, Francipane MG, Medema JP, Stassi G. Colon cancer stem cells: promise of targeted therapy. Gastroenterology 2010; 138:2151-2162.

12 Ito T, Kwon HY, Zimdahl B, et al. Regulation of myeloid leukaemia by the cell-fate determinant Musashi. Nature 2010; 466:765-768

13 Kharas MG, Lengner CJ, Al-Shahrour $\mathrm{F}$, et al. Musashi-2 regulates normal hematopoiesis and promotes aggressive myeloid leukemia. Nat Med 2010; 16:903-908

14 Barbouti A, Höglund M, Johansson B, et al. A novel gene, MSI2, encoding a putative RNA-binding protein is recurrently rearranged at disease progression of chronic myeloid leukemia and forms a fusion gene with HOXA9 as a result of the cryptic $\mathrm{t}(7 ; 17)(\mathrm{p} 15 ; \mathrm{q} 23)$. Cancer Res 2003; 63:1202-1206.

15 Calabretta B, Perrotti D. The biology of CML blast crisis. Blood 2004; 103:4010-4022.

16 Pear WS, Miller JP, Xu L, et al. Efficient and rapid induction of a chronic myelogenous leukemia-like myeloproliferative disease in mice receiving P210 bcr/abl-transduced bone marrow. Blood 1998; 92:3780-3792.

17 Nakahara F, Sakata-Yanagimoto M, Komeno Y, et al. Hes1 immortalizes committed progenitors and plays a role in blast crisis transition in chronic myelogenous leukemia. Blood 2010; 115:2872-2881. 